\title{
Identification of new aberrantly expressed miRNAs in intestinal-type gastric cancer and its clinical significance
}

\author{
XINHUALI ${ }^{1}$, FEIJUN LUO ${ }^{4}$, QIAN LI ${ }^{1}$, MEIHUA XU ${ }^{1}$, DEYUN FENG $^{2}$, GUIYING ZHANG $^{1}$ and WEI WU ${ }^{3}$ \\ Departments of ${ }^{1}$ Gastroenterology, ${ }^{2}$ Pathology, and ${ }^{3}$ Geriatric Surgery, Xiangya Hospital, Central \\ South University, Changsha 410008, Hunan Province, P.R. China; ${ }^{4}$ Department of Pathology, \\ Addenbrooke's Hospital, University of Cambridge, Hills Road, Cambridge, UK
}

Received May 30, 2011; Accepted July 25, 2011

DOI: $10.3892 /$ or.2011.1437

\begin{abstract}
RNAs are small 19 to 22 nucleotide sequences of RNA that negatively regulate gene expression. miRNA expression profiles may become useful biomarkers for diagnostics, prognosis and prediction of response to treat, and it could be a powerful tool for cancer prevention and therapeutics. Several miRNA expression profiles of miRNAs in gastric cancer have been reported, but these studies screened only few miRNAs and samples used in experiments include several different subtypes of gastric cancers, which decrease the sensitivity to identify new aberrant miRNAs. In this study, a miRNA expression profile was identified by miRCURY LNA Array (v.14.0) between intestinal-type gastric cancers and normal tissues. Forty miRNA precursors were up-regulated and thirty-six miRNA were down-regulated in intestinal-type gastric cancers $(\mathrm{p}<0.01)$. Sixteen new miRNAs were found in intestinaltype gastric cancers. Seventeen new miRNAs were found in intestinal-type gastric cancers. miR-145, miR-27a, miR-494 are differently expressed between intestinal-type and diffuse-type gastric cancers. miR-32, miR-182 and miR-143 dysregulated expression levels are related with different pathological stages of intestinal-type gastric cancers $(\mathrm{p}<0.01)$. Taken together, aberrantly expressed miRNAs may offer new clues to tumorigenesis of gastric cancers. miR-32, miR-182 and miR-143 may be potential diagnostic biomarkers for intestinal-type gastric cancers.
\end{abstract}

\section{Introduction}

Gastric cancer is the fourth most common human malignant disease and the second most frequent cause of cancer-related death worldwide, with particularly high frequencies in East

Correspondence to: Professor Wei Wu, Department of Geriatric Surgery, Xiangya Hospital, Central South University, No.88 Xiangya Road, Changsha 410008, Hunan Province, P.R. China

E-mail: luo10000@yahoo.com.cn

Key words: intestinal-type gastric cancer, diffuse-type gastric cancer, miRNA, gene expression
Asia (1). Although significant advances have been achieved since the Human Genomic Project finished, the molecular pathogenesis of gastric cancers still remains to be explored. Markers for tumorigenesis and progression of gastric cancers have not yet been discovered and specific therapeutic targets have not been identified. Biomarker research is focused on a new class of small non-coding RNAs-miRNAs, as several studies showed the superior promise of miRNA expression profiles in tumor classification, diagnosis and prognosis (2). A growing number of direct and indirect evidence suggests a relationship between altered miRNA expression and gastric cancers $(3,4)$.

miRNAs are important factors in tumorigenesis and have been the subject of research in many types of cancers. miRNAs are small 19 to 22 nucleotide sequences of RNA and participate in the regulation of cell differentiation, cell cycle progression and apoptosis. miRNAs are believed to function primarily as negative regulators of gene expression following binding to conserved sequences within the $3^{\prime}$ untranslated region of target mRNAs. While the biological roles of miRNA are under intense investigation, they are believed to define and maintain cellular fate in a manner similar to transcription factors, alterations in miRNA expression may be an important contributor to the development of cancers. miRNAs are also abnormally down-regulated or up-regulated in gastric cancer tissue. Screening aberrant miRNAs may provide a basis for understanding the functional role of miRNAs in cancers (5).

Aberrant expression of miRNAs in gastric cancers, screened by gene chip analysis or reverse transcriptase quantitative PCR (RT-qPCR), has been reported. Katada and colleagues investigated the expression levels of miRNAs in gastric cancers and a set of 72 miRNAs were analyzed. They found that the expression levels of 3 miRNAs (miR-34b, miR-34c and miR-128a) were significantly up-regulated and those of 3 miRNAs (miR-128b, miR-129 and miR-148) were down-regulated in gastric cancer tissues when compared with those of the paired normal tissues (6). Guo and colleagues firstly used microParaflo microfluidic chip to detect the miRNA expression profile in gastric cancers. They found that miR-768-3p, miR-139-5p, miR-378, miR-31, miR-195, miR-497 and miR-133b are significantly decreased in gastric cancers; and miR-20b, miR-20a, miR-17, miR-106a, miR-18a, miR-21, miR-106b, miR-18b, miR-421, miR-340*, miR-19a 
and miR-658 are significantly increased in gastric cancer tissues (7). Ueda and colleagues analyzed 160 paired samples of non-tumor mucosa and cancers, and found that 22 miRNAs were up-regulated and 13 were down-regulated in gastric cancers (8). It was estimated that about 1000 miRNAs exist in human, but less than 500 human miRNA genes were screened in the previous studies. It is necessary to identify new aberrant expression miRNAs in gastric cancers by screening all miRNAs or near 1000 miRNA genes.

Different subtypes of gastric cancers show multiple biological and clinical differences. Genetic and epigenetic events, including 1q loss of heterozygote ( $\mathrm{LOH})$, microsatellite instability and hypermethylation, have mostly been reported in intestinal-type gastric carcinoma and its precursor lesions, whereas $17 \mathrm{p} \mathrm{LOH}$, mutation or loss of E-cadherin are more often implicated in the development of diffuse-type gastric cancer (9). Intestinal-type gastric cancers are related with infection with Helicobacter pylori, and diffuse-type gastric cancers are often absent of infection and have high levels of transforming growth factor $\beta$ (TGF- $\beta$ ) expression $(10,11)$. Aberrant expression of miRNAs are identified by comparing gastric cancer tissues and normal tissues, but the paired samples came from different stages of gastric cancers. Usually investigators set 2.0-fold increase or decrease expression as a cut-off, samples mixed with different subtypes and stages will be neglected in altered miRNAs in the chip analysis.

To resolve the issue, we used a 904-human miRNA gene chip, which is nearly all the miRNAs of human and identified new aberrant expression miRNAs between intestinal-type gastric cancers and normal mucosa. We identified 36 new obviously changed expression miRNAs in intestinal-type gastric cancers. It showed that miR-145, miR-27a and miR-494 are differently expressed between intestinal-type and diffuse-type gastric cancers. miR-182, miR-143 miR-32 and miR-886-5p dysregulated expressions are related with progression of gastric cancers. Our data offer new clues to study the molecular mechanism of carcinogenesis of gastric cancers.

\section{Materials and methods}

Tissue procurement. The tissue samples in this study were derived from patients undergoing a surgical procedure to remove a portion of the gastric cancers at the Xiangya Hospital, Central South University, Changsha, China. The collection of samples conformed to the policies of China and practices of the facility's Institutional Review Board. Upon removal of the surgical specimen, research personnel immediately transported the tissue to the surgical pathology laboratory. Pathology faculty performed a gross analysis of the specimen and selected cancerous appearing gastric tissue and normal appearing gastric tissue for research. Each sample was placed in a cryovial and flash-frozen in liquid nitrogen until analysis. Research samples were confirmed by subsequent pathologic analysis. These slides were examined by one of us (D.F.) to determine if the benign tissues contained any gastric tumor cells. Benign tissues containing residual tumor were excluded from the study.

Total RNA isolation and quality analysis. Frozen tissues $(\sim 10 \mathrm{mg})$ were first pulverized in a stainless steel mortar and pestled. Total RNA was isolated from tissues in $1 \mathrm{ml}$ of TRIzol (Invitrogen, Carslbad, CA) and used RNeasy mini kit (Qiagen) to purify RNA according to the manufacturer's instructions. RNA concentration was determined by analyzing $1 \mu \mathrm{l}$ of solution using the ND-1000 micro-spectrophotometer (NanoDrop Technologies, Wilmington, DE). RNA integrity was evaluated using the Agilent 2100 Bioanalyzer (Agilent Technologies, Palo Alto, CA). RNA integrity number (RIN) was determined using the RIN algorithm of the Agilent 2100 expert software (12).

miRNA precursor expression profiling. Total RNA (30 ng) of 6-paired samples were labeled with $\mathrm{Hy} 5^{\mathrm{TM}}$ or $\mathrm{Hy} 3^{\mathrm{TM}}$ by miRCURY Array Power Labeling kit (Exiqon). Hy5 and Hy3 labeling reactions of every paired sample are combined and hybridized with miRCURY Array LNA microRNA chip (v.14.0) on a hybridization station (Exiqon). After hybridization, scanning is performed with the Axon GenePix 4000B microarray scanner. GenePix pro V6.0 is used to read the raw intensity of the image. The ratio of red signal to green signal was calculated after background subtraction and normalization using the global Lowess (Locally Weighted Scatter plot Smoothing) regression algorithm (MIDAS, TIGR Microarray Data Analysis System), which produced the best within-slide normalization to minimize the intensity-dependent differences between the dyes. Replicated spots on the same slide have been averaged by getting a median ratio of replicated spots. Slide normalization was performed by scale normalization to reduce between-slide variability (13). The statistical significance of differentially expressed miRNAs was analyzed by 2 -fold change and t-test.

miRNA quantification by real-time RT-PCR. SYBR Green RT-qPCR assay was used for miRNA quantification. In brief, $40 \mathrm{ng}$ of total RNA containing miRNA was polyadenylated by poly(A) polymerase and was reversely transcripted to cDNA using miScript Reverse Transcription kit according to the manufacturer's instructions (Qiagen, Valencia, CA, USA). miScript SYBR Green PCR kit was used and miScript Universal primer was provided by the manufacturer (Qiagen), RT-qPCR was performed in ABI PRISM 7900 Real-time PCR system (Applied Biosystems). Each reaction was performed in a final volume of $10 \mu \mathrm{l}$ containing $2 \mu \mathrm{l}$ of cDNA, $0.5 \mathrm{mM}$ of each primer and 1X SYBR Green PCR Master mix (Qiagen). The amplification program was: denaturation at $95^{\circ} \mathrm{C}$ for $10 \mathrm{~min}$, followed by 45 cycles of $94^{\circ} \mathrm{C}$ for $15 \mathrm{sec}, 55^{\circ} \mathrm{C}$ for $30 \mathrm{sec}$ and $70^{\circ} \mathrm{C}$ for $30 \mathrm{sec}$, in which fluorescence was acquired. At the end of the PCR cycles, melting curve analyses were performed as well as electrophoresis of the products on $2.5 \%$ agarose gels in order to validate the specific generation of the expected PCR product. Each sample was run in triplicates for analysis. The expression levels of miRNAs were normalized to RNU6B. Relative gene expression was calculated as $2^{-(C T M i R N A-C T R N U 6 B}$ RNA) . Except reference PCR primers RNU6B (5'-CGC TTC ACG AAT TTG CGT GTC AT-3'), all miRNA PCR assays were purchased from Qiagen Co. miR-1259 (MPH01747A-200), miR-886-3p (MPH01372A-200), miR-145 (MPH00046A-200), miR-125b (MPH00023A-200), miR-143 (MPH01177A-200), hsa-miR-27a (MPH01239A-200), miR-214 (MPH01223A-200), miR-424 (MPH00211A-200), miR-375 

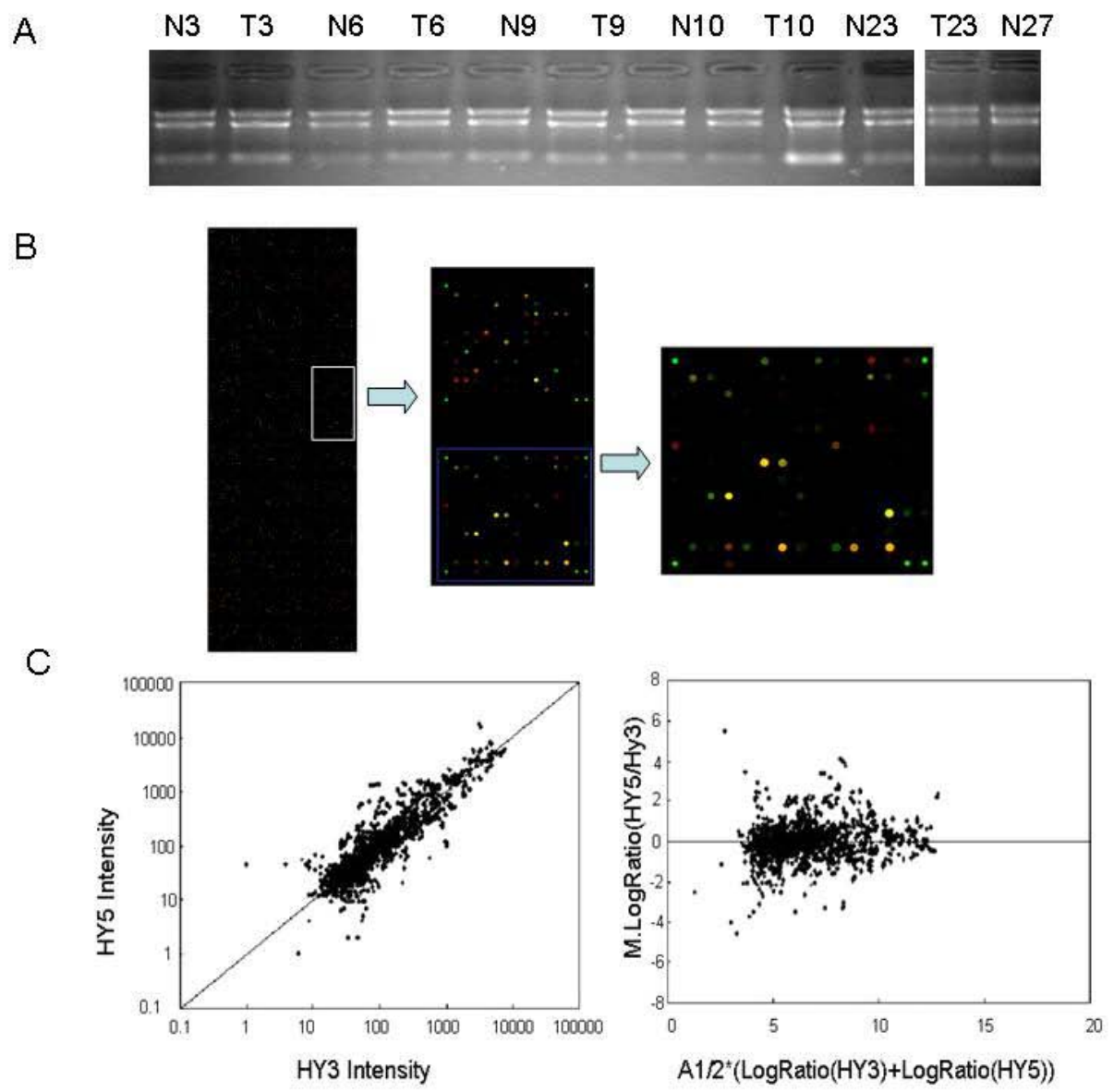

Figure 1. miRCURY LNA array analyzes of miRNA gene expression profile of intestinal-type gastric cancer and normal tissues. (A) Gel electrophoresis check of total RNA from samples. High quality of the RNA isolated from gastric cancers and normal tissues. (B) Part of picture shows hybridization of the miRNA chip. Tumor tissues were labeled with Hy5 and red spots represent up-regulated-expression miRNAs in the gastric cancers. Paired normal tissues were labeled with $\mathrm{Hy} 3$ and green spots represent down-regulated-expression miRNAs in the gastric cancers. (C) MA Plot shows the results after Lowess Normalization. MA plot is especially useful for the detection of the intensity-dependent effects in the log-ratios. Formula of log-ratios and fluorescence intensity $\mathrm{A}=1 / 2(\log 2 \mathrm{~A}+$ $\log 2 \mathrm{~B}), \mathrm{y}$-axis represents the $\log$-ratios $\mathrm{M}=\log 2(\mathrm{~A} / \mathrm{B})$.

(MPH00191A-200), miR-182 (MPH00166A-200), miR886-5p, (MPH00037A-200) (Qiagen).

Hierarchical clustering analysis. Prior to hierarchical clustering, miRNA profiles were standardized to have mean zero and standard deviation one. Clustering analysis was performed with average linkage and Pearson correlation. In brief, the object of this algorithm is to compute a dendrogram that assembles all elements into a single tree. For any set of $n$ miRNA genes, an upper-diagonal similarity matrix is computed by using the metric described above, which contains similarity scores for all pairs of genes. The matrix is scanned to identify the highest value (representing the most similar pair of genes). A node is created joining these two genes, and a gene expression profile is computed for the node by averaging observation for the joined elements (missing values are omitted and the two joined elements are weighted by the number of genes they contain).

Statistical analysis. Results of experiments are expressed as mean \pm SD. Student's unpaired $t$-test was used to compare values of test and control samples. $\mathrm{P}<0.05$ indicated significant difference.

\section{Results}

miRNA expression profiles in intestinal-type gastric cancers. Total RNA was isolated from tissues and the quality of the RNA was analyzed by agarose gel electrophoresis (Fig. 1A). miRCURY Array LNA microRNA chip (v.14.0) was used to evaluate miRNA expression profiles between gastric cancer tissues and adjacent non-tumors tissues. Tumor tissues were labeled with Hy5 and normal tissues were labeled with $\mathrm{Hy} 3$, red spots represent up-regulated miRNAs in the gastric cancers and green spots represent down-regulated miRNAs in the gastric cancers (Fig. 1B). After Lowess Normalization, the miRNA expression profile was identified by statistical analysis (Fig. 1C). All 6 paired gastric cancers were pathologically classified in TNM category stage III or IV. The aberrantly expressed miRNAs are listed in Tables I and II. When setting average change $>2$-fold and $p$-value $<0.01$ as a cut-off level, 40 miRNAs are up-regulated and 36 miRNAs are down-regulated in intestinal-type gastric cancers. Comparing with previous studies of miRNAs and gastric cancers, we found that 24 among 40 genes have been reported in previous publications, for example: miR-21, miR-145, miR-96, miR- 
Table I. List of up-regulated expression miRNAs in intestinaltype gastric cancers.

\begin{tabular}{|c|c|}
\hline Gene name & Fold \\
\hline hsa-miR-1259 & 19.89 \\
\hline hsa-miR-32 & 17.93 \\
\hline hsa-miR-20a & 17.74 \\
\hline hsa-miR-886-5p & 16.85 \\
\hline hsa-miR-21 & 10.44 \\
\hline hsa-miRPlus-A1087 & 9.76 \\
\hline hsa-miR-96 & 8.29 \\
\hline hsa-miR-20a* & 7.87 \\
\hline hsa-miR-182 & 7.43 \\
\hline hsa-miR-17 & 6.85 \\
\hline hsa-miR-18a & 6.51 \\
\hline hsa-miR-19a & 6.30 \\
\hline hsa-miR-886-3p & 6.12 \\
\hline hsa-miR-1308 & 6.05 \\
\hline hsa-miR-106a & 5.98 \\
\hline hsa-miR-335 & 5.81 \\
\hline hsa-miR-18b & 5.01 \\
\hline hsa-miR-223 & 4.90 \\
\hline hsa-miR-27a & 4.72 \\
\hline hsa-miR-183 & 4.30 \\
\hline hsa-miR-203 & 4.27 \\
\hline hsa-miR-362-3p & 4.14 \\
\hline hsa-miR-93 & 3.88 \\
\hline hsa-miR-451 & 3.82 \\
\hline hsa-miR-424 & 3.37 \\
\hline hsa-miR-491-3p & 3.22 \\
\hline hsa-miR-92a & 3.11 \\
\hline hsa-miR-33a & 2.98 \\
\hline hsa-miR-140-5p & 2.94 \\
\hline hsa-miR-29a* & 2.79 \\
\hline hsa-miR-23b & 2.71 \\
\hline hsa-miR-542-3p & 2.65 \\
\hline hsa-miR-19b & 2.61 \\
\hline hsa-miR-141 & 2.60 \\
\hline hsa-miR-200b & 2.40 \\
\hline hsa-miR-107 & 2.31 \\
\hline hsa-miR-1826 & 2.29 \\
\hline hsa-miR-222 & 2.15 \\
\hline hsa-miR-214 & 2.11 \\
\hline hsa-miR-17* & 2.09 \\
\hline
\end{tabular}

$\mathrm{P}<0.01$ and miRNAs in bold fonts have been reported in previous studies of gastric cancers.

106a, and miR-17. New up-regulated genes include recently named genes such as: miR-886-3p, miR-886-5p, miR-1259 and miR-1308. Nineteen genes among 36 down-regulated genes have been reported before such as: miR-16, miR-143, miR-145, miR-375, and miR-378; and 16 new miRNAs were down-regulated in intestinal-type gastric cancers
Table II. List of down-regulated expression miRNA in intestinal-type gastric cancers.

\begin{tabular}{|c|c|}
\hline Gene name & Fold \\
\hline hsa-miR-375 & 0.20 \\
\hline hsa-miR-147b & 0.22 \\
\hline hsa-miR-422a & 0.27 \\
\hline hsa-miR-29c & 0.28 \\
\hline hsa-miR-640 & 0.28 \\
\hline hsa-miR-139-5p & 0.28 \\
\hline hsa-miR-378 & 0.29 \\
\hline hsa-miR-513a-5p & 0.30 \\
\hline hsa-miR-24 & 0.31 \\
\hline hsa-miRPlus-E1033 & 0.37 \\
\hline hsa-miR-145 & 0.37 \\
\hline hsa-miR-494 & 0.37 \\
\hline has-miRPlus-1238 & 0.37 \\
\hline hsa-miR-449a & 0.39 \\
\hline hsa-miR-361-3p & 0.40 \\
\hline hsa-miR-125b & 0.41 \\
\hline hsa-miR-155 & 0.42 \\
\hline hsa-miR-196a* & 0.43 \\
\hline hsa-miR-627 & 0.44 \\
\hline hsa-miR-16 & 0.44 \\
\hline hsa-miR-128 & 0.44 \\
\hline hsa-miR-132 & 0.44 \\
\hline hsa-miR-152 & 0.44 \\
\hline hsa-miR-143 & 0.44 \\
\hline hsa-miR-320b & 0.45 \\
\hline hsa-let-7g & 0.45 \\
\hline hsa-miR-125a-5p & 0.45 \\
\hline hsa-miR-320c & 0.47 \\
\hline hsa-miR-486-5p & 0.48 \\
\hline hsa-miR-433 & 0.48 \\
\hline hsa-miR-320a & 0.48 \\
\hline hsa-miR-101 & 0.49 \\
\hline hsa-miR-194* & 0.49 \\
\hline hsa-miR-191 & 0.49 \\
\hline hsa-miR-30d & 0.50 \\
\hline hsa-miRPlus-E1117 & 0.50 \\
\hline
\end{tabular}

$\mathrm{P}<0.01$ and miRNAs in bold fonts have been reported in previous studies of gastric cancers.

such as: miR-147b, miR-422a, miR-320a, miR-320b and miR-30d were found with down-regulated expression such as: miRPlus-E1033 and miRPlus-E1117. For gene chip analysis, all our samples include node or organ metastasis and also many metastasis-related miRNAs were dysregulated in the gastric cancers, including: $\mathrm{miR}-21$, miR-27b, miR-29a*, miR-19b, miR-222, miR-214, miR-16 and miR-143. We provide new miRNA expression profiles of intestinal-type gastric cancers (Tables I and II). 
A

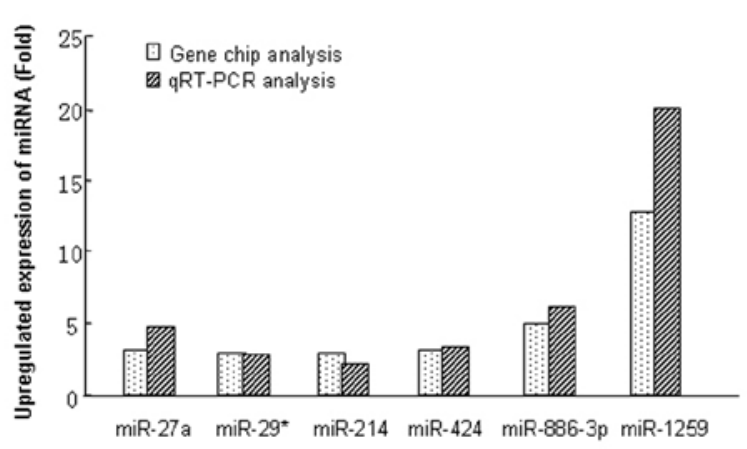

B

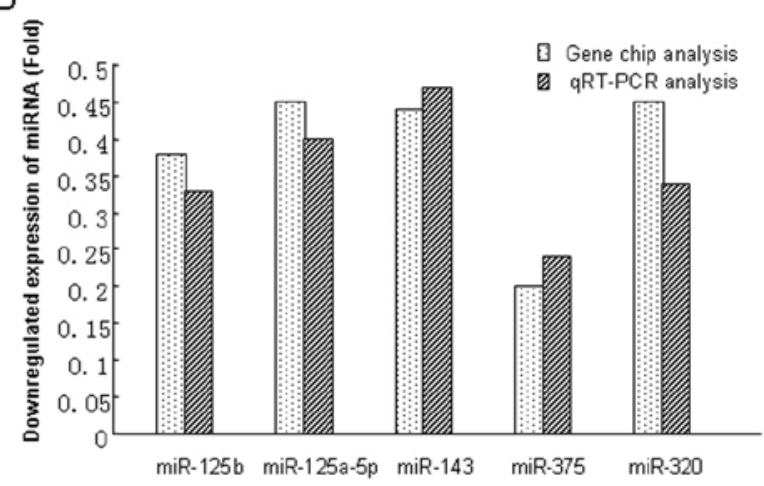

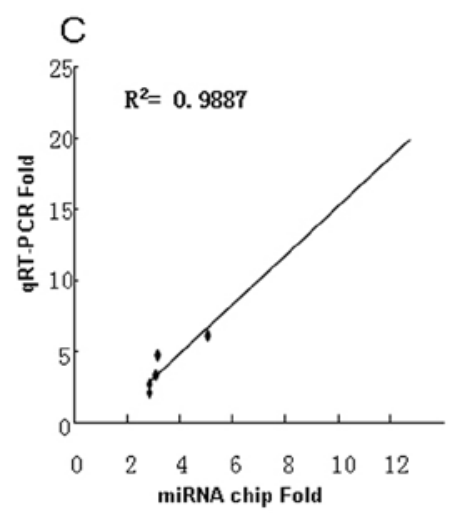
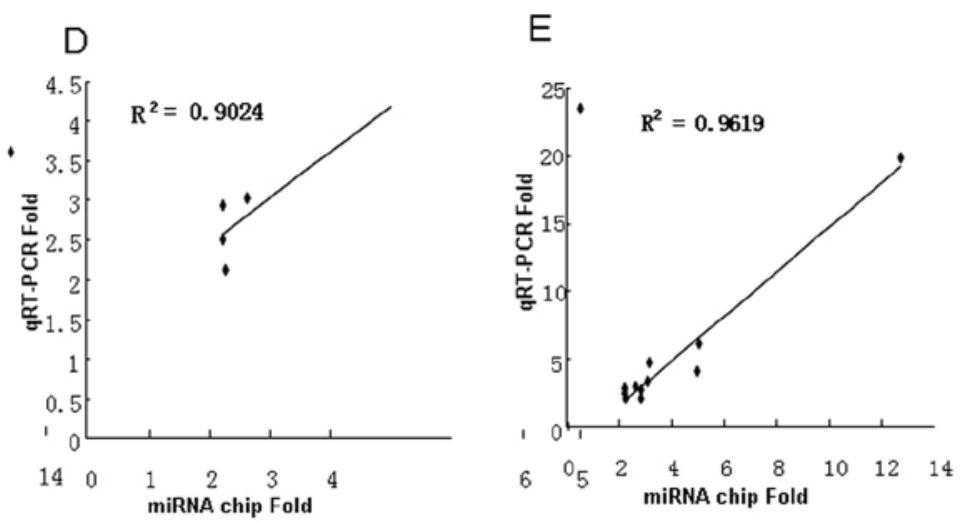

Figure 2. Comparison of miRNA expression levels between chip and qRT-PCR. (A) Comparing up-regulated miRNA expression levels between chip and qRT-PCR in paired intestinal-type gastric cancers. (B) Comparing down-regulated miRNA expression levels between chip and qRT-PCR in paired intestinaltype gastric cancers. (C) Correlation of chip vs. qPCR data for up-regulated miRNAs. (D) Correlation of chip vs. qPCR data for down-regulated miRNAs. (E) Regression plot of the increase or the decrease of miRNAs.

Comparison of miRNA expression level between RT-qPCR and chip analysis. The RT-qPCR system detects PCR products as they accumulate rather than assaying final product concentrations after a fixed number of cycles. The iCycler sequence detection system (Bio-Rad Laboratories, Hercules, CA) provides a way to monitor in real-time the accumulation of DNA synthesized during the PCR process. It excites fluorescence of the selected dye and images the emitted light during each thermal cycle of the PCR run. To compare the relative miRNA expression levels between RT-qPCR and chip analysis, miR-27, miR-32, miR-125a-3p, miR-125b, miR-143, miR-381, miR-214, miR-320b, miR-375, miR-424, miR-886-5p and miR-1259 were selected and they are among the top differentially expressed miRNAs by chip analysis (Tables I and II). We analyzed expression levels of these miRNAs in 70-paired samples of intestinal-type gastric cancer, and results showed that miRNA expression in the paired benign and tumor tissue was consistently increased or decreased in all cases. We found that all 6 up-regulated miRNAs in chip analysis increase expression levels in gastric cancers tissues, and 6 miRNAs down-regulated miRNAs also decrease expression in normal tissues (Fig. 2A and B). Expression levels of 6 up-regulated miRNAs varied with a range from $-34.5 \%$ to $36.1 \%$, respectively. Expression levels of 6 down-regulated miRNAs varied that with a range from $-32.3 \%$ to $16.6 \%$. The correlation of relative miRNA expression levels was analyzed by cDNA array (Array fold) vs. RT-qPCR (qPCR fold). Pearson correlation of down-regulated miRNAs was $0.98(\mathrm{p}<0.01)$ and Pearson correlation of up-regulated miRNAs was $0.90(\mathrm{p}<0.01)$. Pearson correlation of both down- and up-regulated miRNAs was $0.96(\mathrm{P}<0.01)$ (Fig. 2C-E). This suggests that aberrantly expressed miRNAs screened by chip analysis were confirmed by RT-qPCR and the data of chip analysis are reliable.

Clustering analysis of significantly changed genes. For clustering analysis, we set the p-value at $<0.01$ as a cut-off level. Expression level of 40 up-regulated genes and 36 downregulated genes were analyzed by unsupervised hierarchical clustering. Our data show that all 76 miRNAs express similar patterns. The heatmap demonstrates that all these genes changed similarly in the different pairs of gastric cancer tissues, and the same pathological stage samples grouped to the same subcluster (Fig. 3).

Different expression pattern of miRNAs between subtypes of gastric cancers. To test for different expression patterns of miRNAs between intestinal-type and diffuse-type gastric cancers, we selected 4 aberrantly expressed miRNAs of gastric cancers and detected the expression levels of miRNAs from 70-paired intestinal-type and 37-paried diffuse-type gastric cancers. The average age of intestinal-type patients is $57.17 \pm 9.70$ and that of diffuse-type patients is $50.23 \pm 11.35$, significant difference exists between the two subtypes $(p=0.011)$. The gender ratio, and the pathological stages of the 

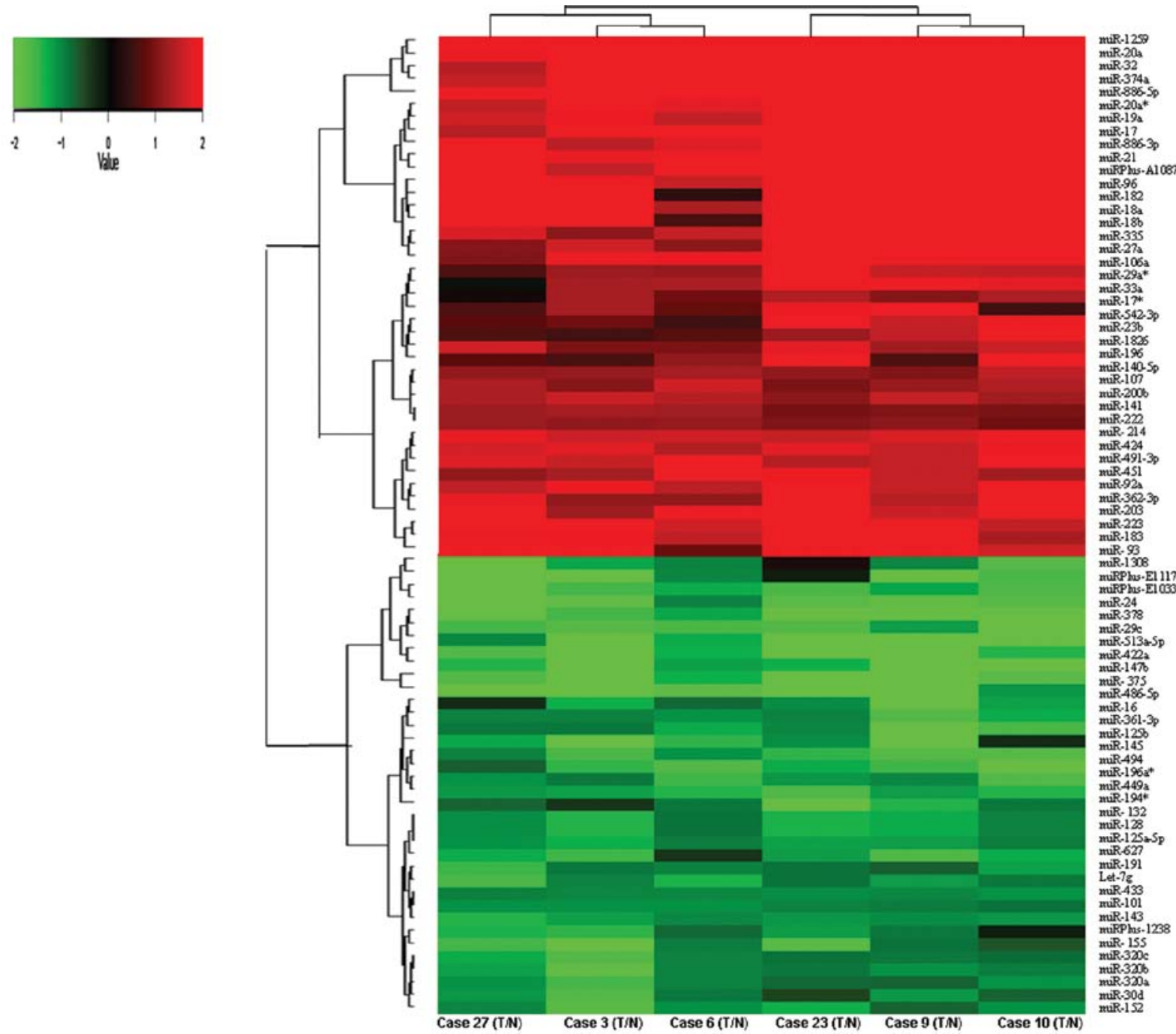

Figure 3. Hierachical cluster analysis of differentially expressed miRNAs in intestinal-type gastric cancers. Comparing with normal tissues, alternation of miRNA expression level in gastric cancers is $>2$-fold $(\mathrm{p}<0.01)$. Comparing with normal tissues, alternation of miRNA expression level in gastric cancers is $>2$-fold $(\mathrm{p}<0.01)$. The heat-map shows accurate classification of the cancers in one subcluster and the normal samples in the other.

two groups do not show statistical difference. Comparing with paired normal tissues, miR-27a expression level increased 2.87-fold in intestinal-type gastric cancers and 4.64-fold in diffuse-type gastric cancers, miR-27a expression levels showed significant difference between the two subtypes $(\mathrm{p}<0.01)$ (Fig. 4A). miR-494 expression was decreased in both subtypes $(\mathrm{p}<0.01)$, but the decrease was 4.39-fold in diffuse-type gastric cancers and only 2.50-fold in intestinal-type, and significant difference also exists between the two subtypes $(\mathrm{p}<0.01)$ (Fig. 4B). miR-145 was down-regulated in multiple cancers and it decreased 4.82-fold in intestinal-type gastric cancers $(\mathrm{p}<0.01)$, but it did not change in diffuse-type gastric cancers ( $p>0.05$ ) (Fig. 4C). On the contrary, miR-145 expression decreased 3.28-fold in intestinal-type gastric cancers and only 2.02-fold in diffuse-type gastric cancers, and there is a significant difference between the subtypes $(\mathrm{p}<0.01)$ (Fig. 4C). Most miRNAs expression levels are similar in both subtype gastric cancers such as miR-886-3p ( $>>0.05)$ (Fig. 4D). The data demonstrate that miRNAs express different pattern between the two subtype cancers and it may result in different pathological progress.

miRNA expression levels are related with progress of intestinal-type gastric cancers. Some miRNAs may target invasion and metastasis-related genes and are involved in the progress. Four dysregulated expression miRNAs are detected in different pathological stages of gastric cancers; comparing with normal tissues, miR-32 expression level of stage $I$ is significantly increased, it is lower than that of stages III and IV in intestinal-type gastric cancers, $p=0.02$ and $p=0.0003$, respectively. Although we did not find a significant difference between stage I and stage II ( $\mathrm{p}=0.06)$, miR-32 expression levels are statistically different between stage II and IV (Fig. 4A). miR-182 expression levels also increased in the different stages of intestinal-type gastric cancers. Significantly difference did not exists between stage I and II or between stage III and IV, but it was significantly different between stage I and II and between stage III and IV (p<0.05) (Fig. 4B). Expression level of miR-143 significantly decreased in stage IV compared 


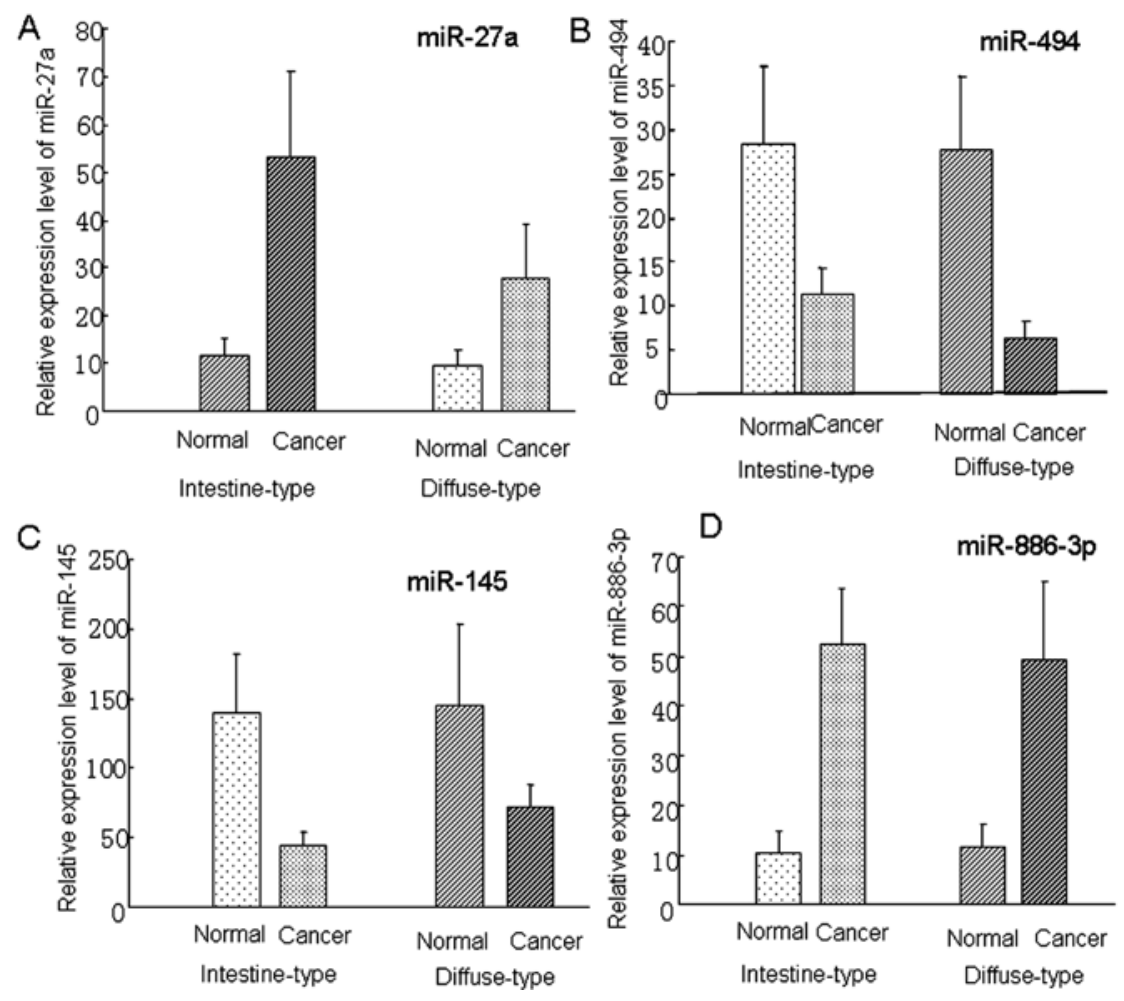

Figure 4. Different expression patterns between diffuse-type and intestinal-type gastric cancers. (A) Real-time PCR analysis of miR-27a in paired diffusetype and intestinal-type gastric cancers. (B) Real-time PCR analysis of miR-494 in paired diffuse-type and intestinal-type gastric cancers. (C) Real-time PCR analysis of miR-145 in paired diffuse-type and intestinal-type gastric cancers. (D) Real-time PCR analysis of miR-886-3p in paired diffuse-type and intestinal-type gastric cancers.
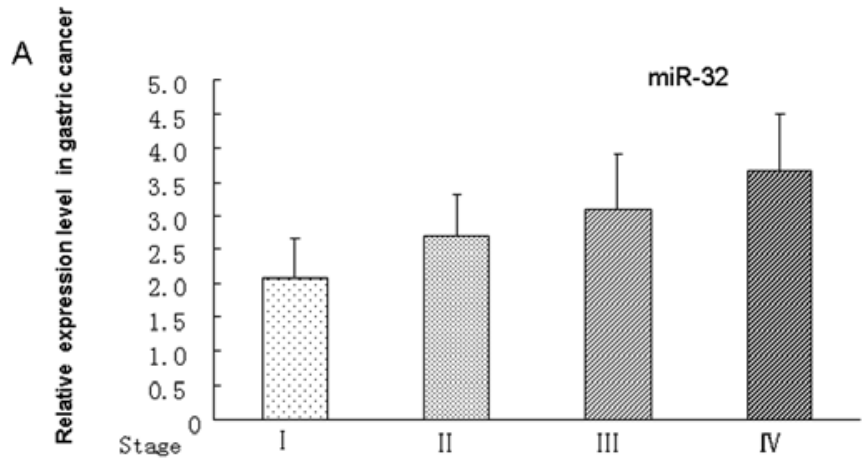

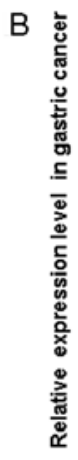

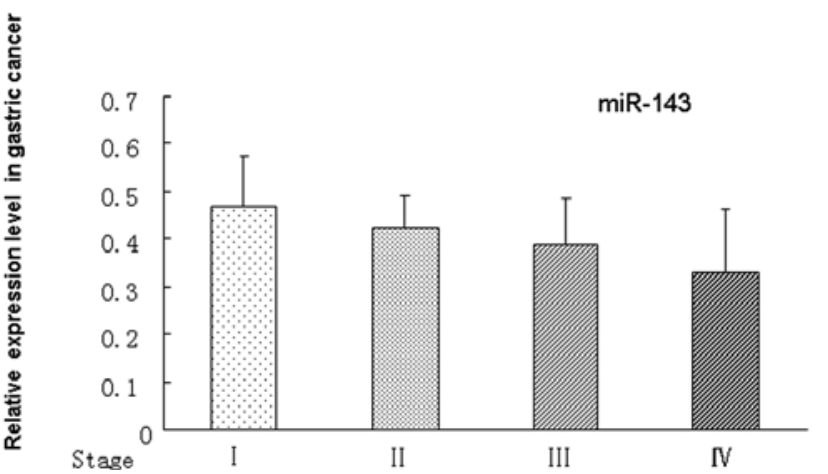

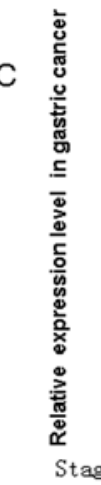

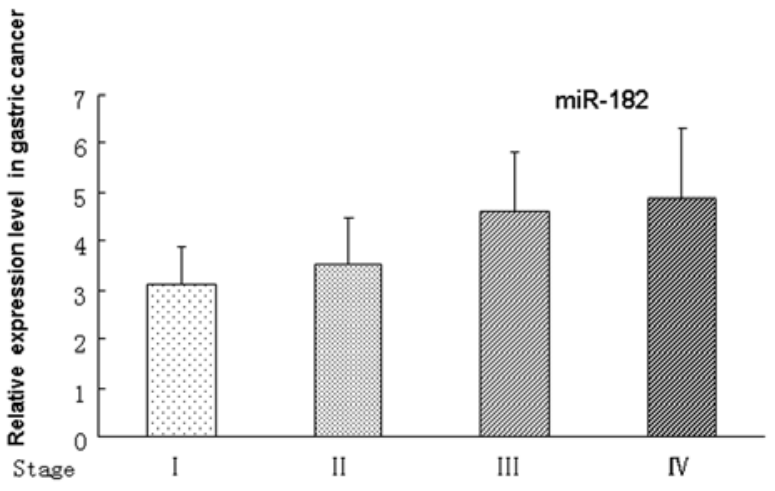

D

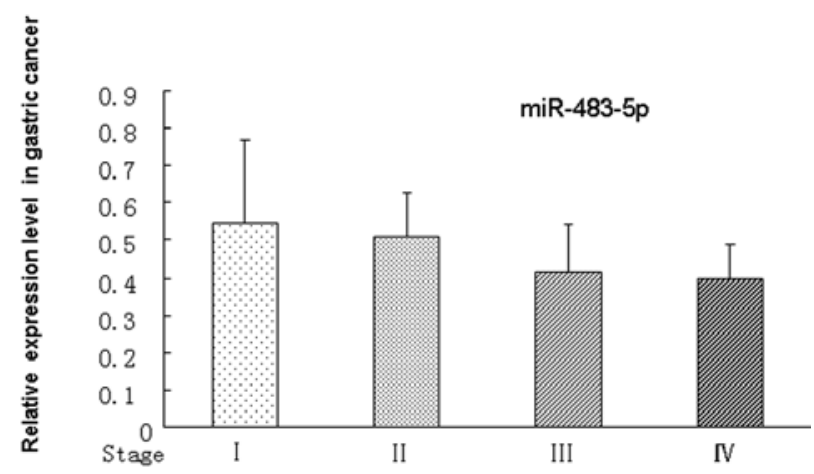

Figure 5. miRNA expression level is associated with progression of intestinal-type gastric cancers. Stage I, 19 cases; stage II, 26 cases; stage III, 21 cases; stage IV, 14 cases. (A) Comparison of miR-32 expression levels in the different stages of intestinal-type gastric cancers (I vs. II, value 0.06; I vs. III, p-value 0.02; I vs. IV, p-value 0.0003; II vs. III, p-value 0.11; II vs. IV, p-value 0.007; III vs. IV, p-value 0.12). (B) Comparison of miR-182 expression levels in the different stages of intestinal-type gastric cancers (I vs. II, p-value 0.20 ; I vs. III, p-value 0.005 ; I vs. IV, p-value 0.005 ; II vs. III, p-value 0.02 ; II vs. IV, p-value 0.02; III vs. IV, p-value 0.35). (C) Comparison of miR-143 expression levels in the different stages of intestinal-type gastric cancers (I vs. II, p-value 0.25; I vs. III, p-value 0.10; I vs. IV, p-value 0.04; II vs. III, p-value 0.14; II vs. IV, p-value 0.03; III vs. IV, p-value 0.22). (D) Comparison of miR-886-5p expression levels in the different stages of intestinal-type gastric cancers (I vs. II, p-value 0.28 ; I vs. III, p-value 0.11 ; I vs. IV p-value 0.08 ; II vs. III, p-value 0.11 ; II vs. IV, p-value 0.11; III vs. IV, p-value 0.04). 
with stage I and II, $\mathrm{p}=0.04$ and $\mathrm{p}=0.03$, respectively $(\mathrm{p}<0.05)$ (Fig. 4C). miR-886-5p expression level was also decreased in the intestinal-type gastric cancers, but no statistical difference exists between stages I and III ( $>0.05)$ or between stage I and IV ( $p>0.05$ ) (Fig. 4D). Taken together, our data showed that miR-32, miR-182 and miR-143 expression levels are related with different pathological subtype of gastric cancers.

\section{Discussion}

Two distinct histological types of gastric cancer show multiple differences in their epidemiology, pathogenesis, genetic profile and clinical outcome. The pathogenesis of intestinal-type gastric cancers follows a multistep progression that usually is initiated by $H$. pylori infection. A wide range of genetic and epigenetic abnormalities including point mutation, loss of heterozygosity, microsatellite instability, and hypermethylation are described in intestinal-type gastric cancers and its precursor lesions. In contrast to the intestinaltype, diffuse-type gastric cancers are defined by a lack of precursor lesions; mutation or epigenetic silencing of the E-cadherin gene appears to be the key carcinogenic event. Accumulating evidence suggests that mRNA and protein expression levels show significant difference in various histological subtypes of gastric cancers (14-16). Reported here are the results of the first detailed miRNA expression profiling study in intestinal-type gastric cancers. Expression profiling identified a large number of miRNAs that are aberrantly expressed in gastric cancers and never report before. Comparing samples mixed with histopathology subtype of gastric cancers, we identified more dysregulated expression miRNAs than any other previous study. It suggests that it is more sensitive to detect new aberrant miRNAs using one subtype and the same or similar stage cancers. Our data showed higher alteration of miRNA expression levels. Usually in miRNA expression 2-fold cut-off is used, and it may neglect aberrant miRNAs in the mixed samples of different subtypes and stages. We grouped the subtype cancers to analyze the different expressions and to resolve part of the issue.

It is more suitable to explore carcinogenesis mechanism of gastric cancers by different subtype of histopathology. miRNA expression pattern of intestinal-type gastric cancers are like that of diffuse-type gastric cancers, but some miRNAs showed different expression between them. Most of miRNAs in gastric cancers may be dysregulated as those in diffuse-type gastric cancers, but some miRNAs expression levels may show great difference or even be contrary. miRNAs expressing different patterns of subtype cancers will increase our understanding of pathogenesis of intestinaltype gastric cancer.

Expression levels of 4 miRNAs were analyzed between two subtypes of gastric cancer. Our data showed different expression patterns and expression levels. miR-27a up-regulated expression in multiple tissues. Ma and colleagues discovered that the 3'UTR of Sprouty2 (Spry2) carried a putative miR-27a binding site, and the Spry2 protein, which has a low expression level in pancreatic adenocarcinoma (17). miR-27a were observed to be highly expressed in MCF-7 breast cancer cells, in which the level of FOXO1 protein is very low. Antisense inhibitors to each of these microRNAs led to a significant increase in endogenous FOXO1 expression (18). The expression of pFOXO1A was inversely correlated with the intestinal type by Lauren's classification, lymphatic invasion and lymph node metastasis (19). Our data show higher level miR-27a expression in diffuse-type gastric cancer, and different expression level of miR-27a may be related with different invasion ability of the two subtypes of gastric cancers. miR-494 target the antiapoptotic protein FGFR2 (20). FGFR2 expression levels are related with the two subtypes of gastric cancers (21). Our data showed miR-494 expression level decreased more in the diffuse-type gastric cancer. It suggests miRNA expression pattern may be involved in the different phenotype of subtype gastric cancers.

According to our data, miR-32 is one of the most overexpression miRNAs and is related with pathological stages in intestinal-type gastric cancers. miR-32 expression vector transfected in human multiple myeloma (MM) cell lines and xenograft studies showed that miR-32 expression resulted in significant suppression of tumor growth in nude mice (22). Ambs and colleagues found that miR-32 directly target Bim and drive the development and progression of prostate cancer (23).

Recent study reported that expression levels of miR-143 was decreased in gastric cancers, and it increased sensitivity to 5-fluorouracil following transfection with miR-143 (24), but whether its expression levels are related with clinical pathology stages in gastric cancer is unknown. Our data showed that miR-143 also belong to metastamir in intestinal-type gastric cancers. The results of $\mathrm{Xu}$ and colleague support our conclusion, showing that miR-143 decreases prostate cancer cell proliferation and migration and miR-143 may repress metastasis-related KRAS gene (25). It suggests miR-32, miR-182 and miR-143 may be potential molecular markers for classifying stages of gastric cancers.

We also found some new aberrant expression miRNAs in gastric cancers such as: miR-886-5p, miR-1259, miR-486-5p, miRPlus-E1033, and miR-1826, which have no or very few reports of aberrant expression in any other cancers. These new aberrant miRNAs are highly expressed in gastric cancer and may regulate mRNAs, and the impact on gene expression in gastric cancer. As more miRNAs are identified and validated, the role of aberrant miRNA expression in gastric cancer will be better understood. Our data may provide diagnostic biomarkers for gastric cancer and offer new molecular targets for therapy of gastric cancer.

\section{Acknowledgements}

We thank KangChen Bio-tech for providing the excellent equipment and technique support and Dr Rifat Hamoudi from Department of Pathology, University of Cambridge for data analysis. This study is supported by National Natural Science Foundation of China (no. 30271516, no. 30800518/H1617 and no. 81072038/H1617).

\section{References}

1. Jemal A, Murray T, Ward E, Samuels A, Tiwari RC, Ghafoor A, Feuer EJ and Thun MJ: Cancer statistics. CA Cancer J Clin 55: 10-30, 2005. 
2. Lu J, Getz G, Miska EA, Alvarez-Saavedra E, Lamb J, Peck D, Sweet-Cordero A, Ebert BL, Mak RH, Ferrando AA, Downing JR, Jacks T, Horvitz HR and Golub TR: MicroRNA expression profiles classify human cancers. Nature 435: 834-838, 2005.

3. Song B and Ju J: Impact of miRNAs in gastrointestinal cancer diagnosis and prognosis. Expert Rev Mol Med 12: E33-E40, 2010.

4. Wang J, Wang Q, Liu H, Hu B, Zhou W and Cheng Y: MicroRNA expression and its implication for the diagnosis and therapeutic strategies of gastric cancer. Cancer Lett 297: 137-143, 2010.

5. Yang L, Belaguli N and Berger DH: MicroRNA and cologastric cancer. World J Surg 33: 636-646, 2009.

6. Katada T, Ishiguro H, Kuwabara Y, Kimura M, Mitui A, Mori Y, Ogawa R, Harata K and Fujii Y: microRNA expression profile in undifferentiated gastric cancer. Int J Oncol 34: 537-542, 2009.

7. Guo J, Miao Y, Xiao B, Huan R, Jiang Z, Meng D and Wang Y: Differential expression of microRNA species in human gastric cancer versus non-tumorous tissues. J Gastroenterol Hepatol 24 652-657, 2009

8. Ueda T, Volinia S, Okumura H, Shimizu M, Taccioli C, Rossi S, Alder H, Liu CG, Oue N, Yasui W, Yoshida K, Sasaki H, Nomura S, Seto Y, Kaminishi M, Calin GA and Croce CM: Relation between microRNA expression and progression and prognosis of gastric cancer: a microRNA expression analysis. Lancet Oncol 11: 136-146, 2010.

9. Nobili S, Bruno L, Landini I, Napoli C, Bechi P, Tonelli F, Rubio CA, Mini E and Nesi G: Genomic and genetic alterations influence the progression of gastric cancer. World J Gastroenterol 17: 290-299, 2011

10. Komuro A, Yashiro M, Iwata C, Morishita Y, Johansson E, Matsumoto Y, Watanabe A, Aburatani H, Miyoshi H, Kiyono K, Shirai Y, Suzuki HI, Hirakawa K, Kano MR and Miyazono K: Diffuse-type gastric carcinoma: progression, angiogenesis, and transforming growth factor $\beta$ signaling. J Natl Cancer Inst 101: 592-604, 2009.

12. Imbeaud S, Graudens E, Boulanger V, Barlet X, Zaborski P, Eveno E, Mueller O, Schroeder A and Auffray C: Towards standardization of RNA quality assessment using user-independent classifiers of microcapillary electrophoresis traces. Nucleic Acids Res 33: E56-E67, 2005.

13. Yang YH, Dudoit S, Luu P, Lin DM, Peng V, Ngai J and Speed TP: Normalization for cDNA microarray data: a robust composite method addressing single and multiple slide systematic variation. Nucleic Acids Res 30: E15-E24, 2002.
14. Hamilton JP and Meltzer SJ: A review of the genomics of gastric cancer. Clin Gastroenterol Hepatol 4: 416-425, 2006.

15. Stock $\mathrm{M}$ and Otto F: Gene deregulation in gastric cancer. Gene 360: 1-19, 2005.

16. Tahara E: Genetic pathways of two types of gastric cancer. IARC Sci Publ 157: 327-349, 2004.

17. Ma Y, Yu S, Zhao W, Lu Z and Chen J: miR-27a regulates the growth, colony formation and migration of pancreatic cancer cells by targeting Sprouty2. Cancer Lett 298: 150-158, 2010.

18. Guttilla IK and White BA: Coordinate regulation of FOXO1 by miR-27a, miR-96, and miR-182 in breast cancer cells. J Biol Chem 284: 23204-23216, 2009.

19. Kim JH, Kim MK, Lee HE, Cho SJ, Cho YJ, Lee BL, Lee HS Nam SY, Lee JS and Kim WH: Constitutive phosphorylation of the FOXO1A transcription factor as a prognostic variable in gastric cancer. Mod Pathol 20: 835-842, 2007.

20. Wang X, Zhang X, Ren XP, Chen J, Liu H, Yang J, Medvedovic M, $\mathrm{Hu} \mathrm{Z}$ and Fan GC: MicroRNA-494 targeting both proapoptotic and antiapoptotic proteins protects against ischemia/reperfusioninduced cardiac injury. Circulation 122: 1308-1318, 2010.

21. Katoh M: Genetic alterations of FGF receptors: an emerging field in clinical cancer diagnostics and therapeutics. Expert Rev Anticancer Ther 10: 1375-1379, 2010.

22. Pichiorri F, Suh SS, Ladetto M, Kuehl M, Palumbo T, Drandi D, Taccioli C, Zanesi N, Alder H, Hagan JP, Munker R, Volinia S, Boccadoro M, Garzon R, Palumbo A, Aqeilan RI and Croce CM: MicroRNAs regulate critical genes associated with multiple myeloma pathogenesis. Proc Natl Acad Sci USA 105: 12885-12890, 2008

23. Ambs S, Prueitt RL, Yi M, Hudson RS, Howe TM, Petrocca F, Wallace TA, Liu CG, Volinia S, Calin GA, Yfantis HG, Stephens RM and Croce CM: Genomic profiling of microRNA and messenger RNA reveals deregulated microRNA expression in prostate cancer. Cancer Res 68: 6162-6170, 2008.

24. Takagi T, Iio A, Nakagawa Y, Naoe T, Tanigawa N and Akao Y: Decreased expression of microRNA-143 and -145 in human gastric cancers. Oncology 77: 12-21, 2009.

25. Xu B, Niu X, Zhang X, Tao J, Wu D, Wang Z, Li P, Zhang W, Wu H, Feng N, Wang Z, Hua L and Wang X: miR-143 decreases prostate cancer cells proliferation and migration and enhances their sensitivity to docetaxel through suppression of KRAS. Mol Cell Biochem 50: 207-213, 2011. 